\title{
Wind Energy Integration into 380 kV System - Impact on Power Quality of MV and LV Networks
}

\author{
E. Vilchez ${ }^{1}$ J. Stenzel ${ }^{2}$ \\ ${ }^{1}$ Department of Power System Control \\ Technische Universität Darmstadt \\ Landgraf-Georg-Str. 4, 64283 Darmstadt, (Germany) \\ Tel.+49(0)615116-5151. Fax.+49(0)6151164259, E-Mail: elda.vilchez@eev.tu-darmstadt.de \\ ${ }^{2}$ Head of Department of Power System Control \\ Technische Universität Darmstadt \\ Landgraf-Georg-Str. 4, 64283 Darmstadt, (Germany) \\ Tel.+49(0)615116-2852. Fax.+49(0)6151164259, E-Mail: juergen.stenzel@eev.tu-darmstadt.de
}

\begin{abstract}
The amount of electrical energy produced by wind mills is constantly increasing. Nowadays detailed analyses considering the impact of wind energy integration on the transmission system are required. The goal of this study is to examine the power quality behavior of the network in case of integration of different amounts of wind energy at different locations of the $380 \mathrm{kV}$ transmission system. In order to analyse the impact of wind energy integration on the network's power quality, a $380 \mathrm{kV}$ transmission system with $\mathrm{MV}$, and LV subsystems is modeled, integrated wind farms are used and power quality analyses are performed.
\end{abstract}

\section{Key words}

Wind energy integration, modelling of a $380 \mathrm{kV}$ transmission system with $\mathrm{MV}$, and LV subsystems, aggregated wind farms, power quality analysis

\section{Introduction}

Wind power is now the world's fastest growing electrical energy source. Germany's long-term aim, for example, is to produce $25 \%$ of the country's electricity from wind power plants by 2025 [1]. With the increase of the electricity production from wind power plants, issues regarding the resulting impact of the integration of this green energy into the power grid are becoming more and more important.

The integration of big wind farms will create new problems regarding the power quality. The power infeed of these renewable energy sources is fluctuating and hardly predictable. Consequently the impact of these power sources not only to the transmission system, but also to the medium and low voltage subsystems, has to be analysed. The goal of this study is to examine the effect of the integration of wind energy to $380 \mathrm{kV}$ and to analyse the impact on the power quality on Medium Voltage (MV) and Low Voltage (LV) system.

In this framework a model of a $380 \mathrm{kV}$ transmission system and wind farms has been modelled and analysed with the help of a commercial power system planning simulation software [2]. The standard EN 50610 [3] and other new regulations have been taken into account for the model.

\section{Network Modeling}

In order to present a general overview of the network a block design of the whole power system model has been created (Fig 1).

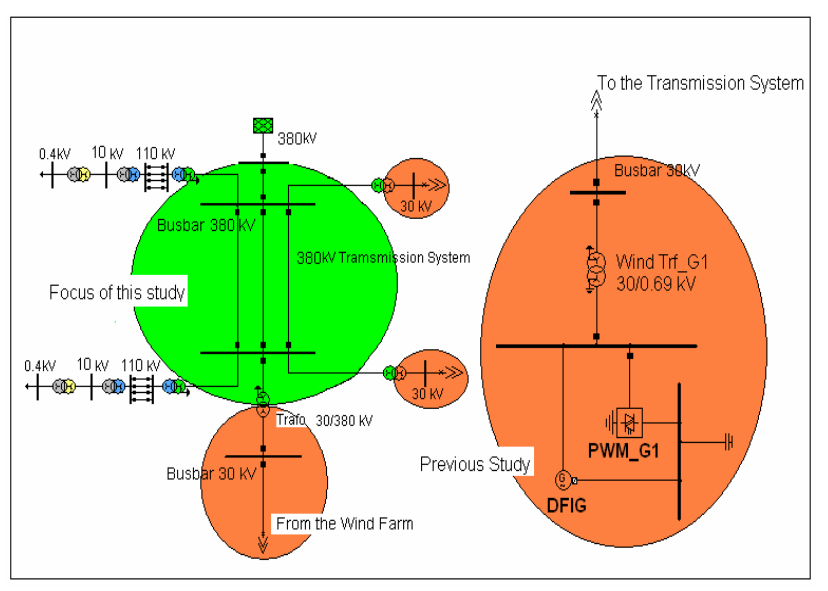

Fig. 1. Block design of the power system model

The block design contains the following significant parts that configure the network:

- $\quad$ The $380 \mathrm{kV}$ transmission system

- The wind farms

- $\quad$ MV and LV system

These blocks will be explained subsequently. 


\section{A. The $380 \mathrm{kV}$ Transmission System}

The first step of this research was the creation of a base model of a $380 \mathrm{kV}$ transmission system.

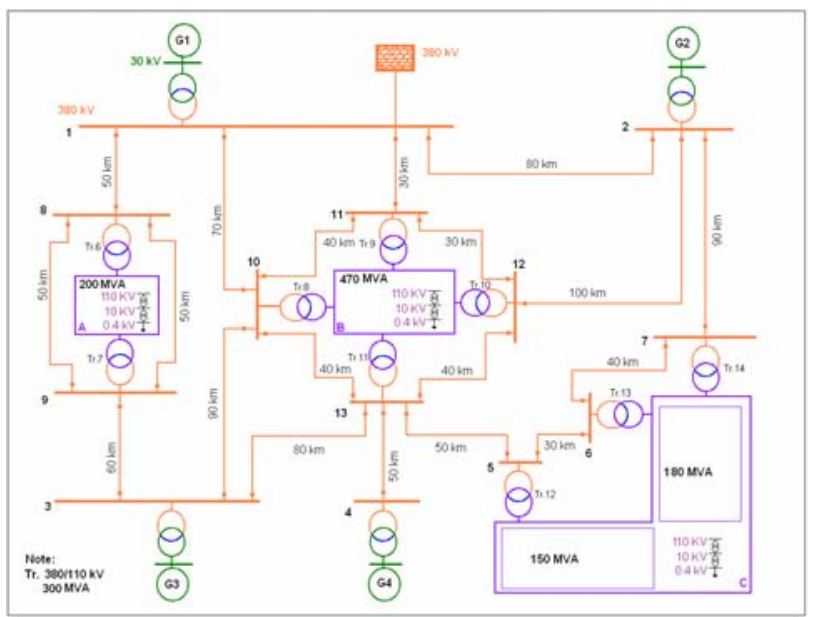

Fig. 2. The $380 \mathrm{kV}$ Transmission System Model

The $380 \mathrm{kV}$ transmission system is a balanced network with 850-km three-phase overhead lines, a connection to one external grid and four different power plants connected to $380 \mathrm{kV}$ substations. The subsystems, 110 $\mathrm{kV}, 10 \mathrm{kV}$ and $0.4 \mathrm{kV}$, are fed from this grid. Three load blocks, which represent the load centres, have been designed. The total load of the system is about 1000 MVA. Each load block has a different configuration, which will be explained afterwards, in order to test different concepts.

\section{B. The wind farms}

It was assumed for the model formation that the wind farms shall have a standard size of 125 MVA. From one to four wind farms are combined to form one big power plant to be interconnected to the $380 \mathrm{kV}$ system.

The wind farms used in this research were based on the doubly-fed induction generator concepts (DFIG, Fig. 3). The main components of the DFIG are: induction generator model, PWM converters and pitch control. Furthermore, it is assumed that the wind-farms are equipped with low-voltage ride-through capability and reactive current support according to the connection standards [5].

It is proven that wind power stations of the same type of doubly-fed asynchronous generators can be clustered to an equivalent power plant when assuming that every wind mill will have a rated power of about 5 MVA [6].

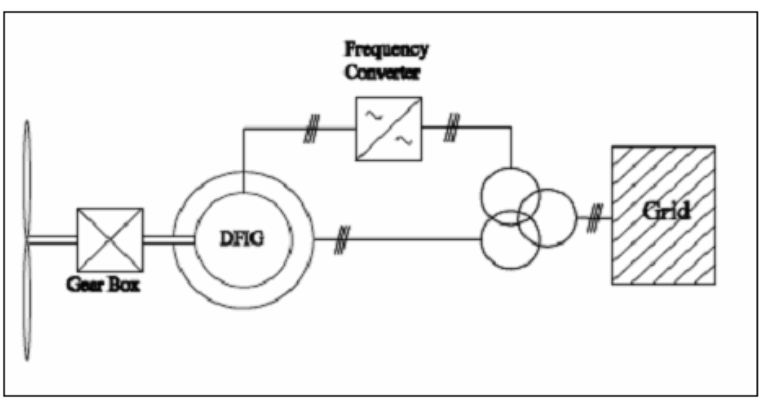

Fig. 3. Doubly-Fed-Induction-Generator

\section{The Aggregated Wind farms}

To reduce the calculation time of the transient simulations, the number of machines representing the wind farm can be reduced without loosing result accuracy for power quality studies. This is done by using an aggregation technique [7].

For this study, aggregated wind farm models have been designed depending on the amount of power of conventional power plants to be substituted by DFIG wind generators. The aggregated wind farm models consist of only one equivalent wind turbine including the detailed controller models as well as the representation of the mechanical system.

The equivalent cable impedance between the aggregated machines and the connection point is defined in a way that the transient short-circuit contribution of the aggregated model at the point of common coupling is equal to the short-circuit contribution of the complete wind farm model.

For validating the approach of using equivalent wind farm models, the response of both the aggregated and the complete model to an unsymmetrical two phase to ground fault at the connection point has been compared [6]. Therefore the model can be reduced and the computation effort can be limited.

\section{The MV and LV System}

The subsystems, $10 \mathrm{kV}$ and $0.4 \mathrm{kV}$, are fed from the 110 $\mathrm{kV}$ transmission grid which is directly connected with the $380 \mathrm{kV}$ system. Three load blocks have been designed. The total load of the system is about 1000 MVA. Each load block has a different configuration in order to test different concepts.

Load Block A shows a central fed system with a total consumption of 200 MVA active power. Two step-down transformers connecting the $110 \mathrm{kV}$ subsystem with the $380 \mathrm{kV}$ level. The MV and LV systems served by stepdown transformers are represented by one busbar each with concentrated loads (Fig.4). 


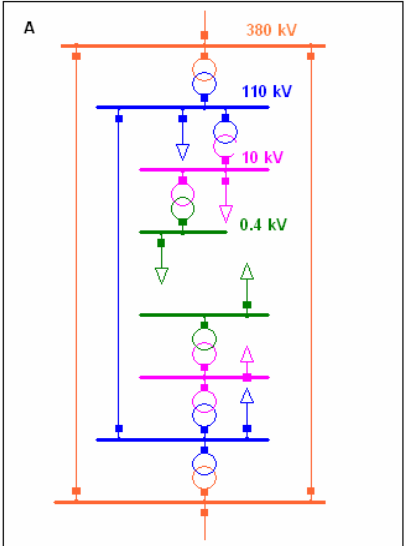

Fig. 4. Load Block Model A

Load Block B shows a peripherally fed system. The areas supplied have a maximum load of 470 MVA respectively. It is connected in four points with the 380 $\mathrm{kV}$ transmission system and the MV and LV systems are supplied by the $110 \mathrm{kV}$ network (Fig. 5).

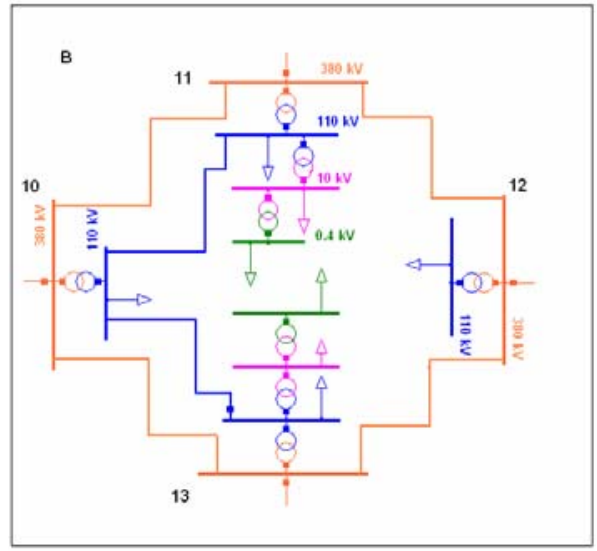

Fig. 5. Load Block Model B

Load Block C has a load consumption of 330 MVA. It is connected in three points with the $380 \mathrm{kV}$ transmission system through the $110 \mathrm{kV}$ network to the MV and LV system (Fig. 6).

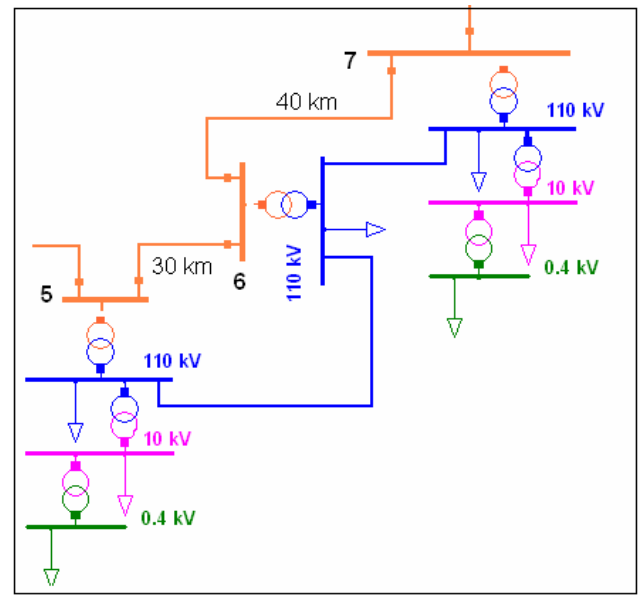

Fig. 6. Load Block Model C

\section{Power Quality Analysis}

For the power quality analysis different scenarios have been created. To keep the computational effort as low as possible and taking into account some software limitation, each load block is analysed separately while the other two blocks are represented by equivalent loads. In this paper load block A is analysed.

A base case is created to show the behaviour of the system without wind energy, with the purpose to make comparisons and analyse the impact of wind integration on the system. The base case is modified and several cases, where the amount of wind energy is changed, have been designed.

The scenarios investigated are:

"Base Case": All generators are conventional synchronous generators directly connected to the transmission system. Load block A is analysed (Fig. 7). This base case scenario represents the system without wind energy. This scenario is used to compare the characteristic of the system without wind energy for this reason is used as base case in the other two scenarios.

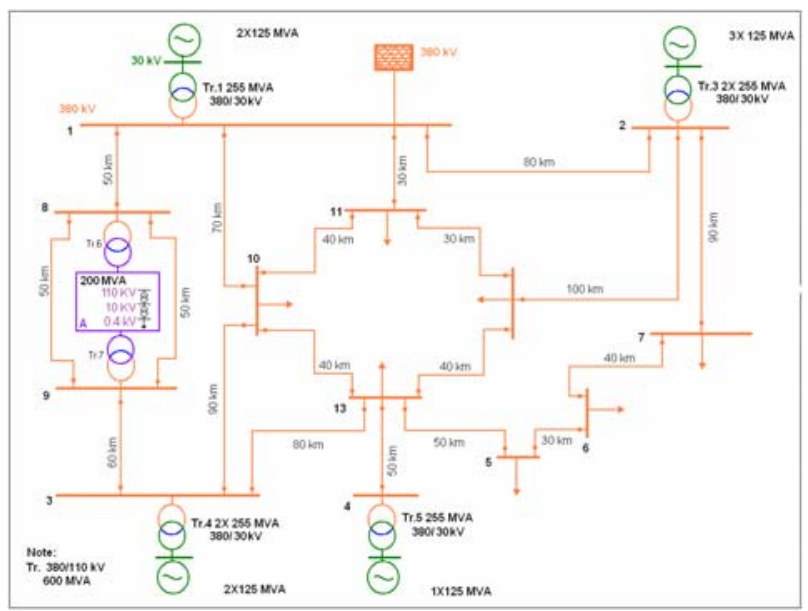

Fig. 7. Base Case Scenario

For the power quality analysis of the following two scenarios, 4 different cases where the amount of wind energy (WE) is changed are studied: 100\% WE, 75\% WE, 50\% WE and 30\% WE. Load block A is analysed.

"Scenario1_WFA": Shows the base model configuration with wind energy integration. The synchronous generators are replaced by wind power plants with the following configuration: power plant 4 is made of one wind farm of $125 \mathrm{MW}$, two WF for power plant 1 and 3 and three WF for power plant 2.

"Scenario2_WFB" As in scenario 1, the synchronous generators are replaced by wind power plants. However in this case the location of one of the WF is altered from power plant 1 to 3 . Load Block A is analysed. 


\section{Voltage Sags Analysis}

The voltage sags analysis uses fault statistics to describe the frequency of errors and uses these statistics to weight the result of each event and calculate the overall effect of failures. Through this foundation sags assessment tables for each scenario were calculated, the results showed the low penetration of voltage sags and these results were similar for the three scenarios and the different cases. This was expected as the low-voltage ride-through capability reduces the effects of voltage sags on the network. Therefore these results where not further discussed.

\section{Harmonic Analysis}

A harmonic analysis is performed using the simulation software [3]. A harmonic penetration was assessed in the $380 \mathrm{kV}$ transmission networks with only one harmonic source on the load 4 from the transmission side and other harmonic sources on the PWM from the wind farm. The $\mathrm{HD} \%$ on the MV $(10 \mathrm{kV})$ and $\mathrm{LV}(0.4 \mathrm{kV})$ is analysed at this point.

The European standard EN 50160 [4] specifies $8 \%$ as the limit for the total harmonic voltage distortion (THD \%) on the supply voltage. The THD \% was measured on the $\mathrm{MV}$ and LV substations (St) and the subsequent results on the St36 and St29 station are shown in figure 8 below.
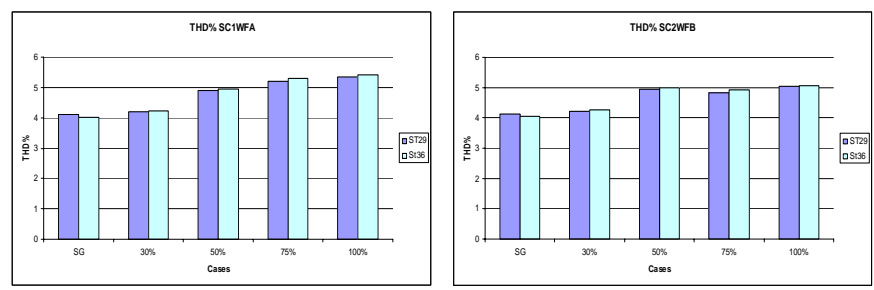

Fig. 8. Total harmonic distortion "Scenario1_WFA" and "Scenario2_WFB"

Here we can see two trends: first in "Scenario1_WFA" the maximum percentage of THD is $5.23 \%$ and $5.22 \%$ on the St 29 and St 36 respectively. These two maximum values are present when $100 \% \mathrm{WE}$ is integrated in the system. This is of interest as the results show an increase of the THD\% when more wind energy is integrated. It is proven that inclusive with $100 \%$ wind energy integration the system is working within the allowed limits since the maximum THD percentage stays below the EN 50160 limit of $8 \%$. Second, related with "Scenario2_WFB", a similar trend to the trend in "Scenario1_WFA" is detected but with the difference that on the $100 \%$ case. the maximum percentage of the THD is $5.03 \%$ and $5.05 \%$ on the St 29 and St 36 respectively. These results show that "Scenario2_WFB2" has less total harmonic distortion. Based on this it is assumed that this scenario has better accuracy than Scenario1_WFA.

In order to examine the behavior of the system in both branches of the "Load Block A" the HD \% on the St 29 and St 31 (MV 10kV) and St 34 and St 36 (LV 0.4 kV) were analysed.

\section{A. "Scenario1_WFA":}

Figure 9 shows the results of the MV and LV system:

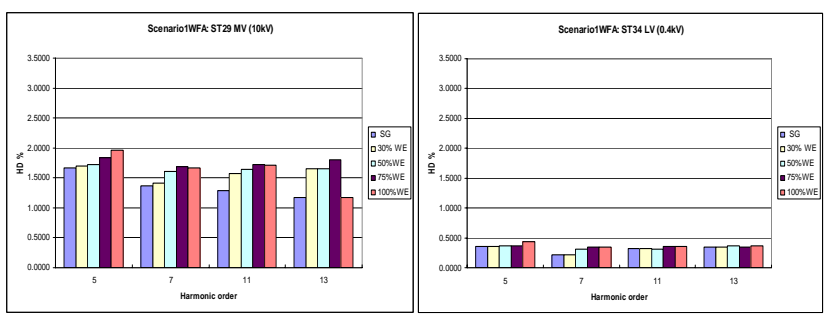

Fig. 9. Harmonic distortion on MV and LV Side "Scenario1_WFA"

The dominating distortion has been identified as the $5^{\text {th }}$ harmonics whereas uncharacteristic distortions of the $11^{\text {th }}$ and $13^{\text {th }}$ harmonics are present. The HD percentage is different in the different cases but with a proportional increase when the system has different amount of wind energy. The results of the harmonic distortion are within the boundaries of the IEEE 519-1992 Harmonic Limits [8].

\section{B. "Scenario2 WFB":}
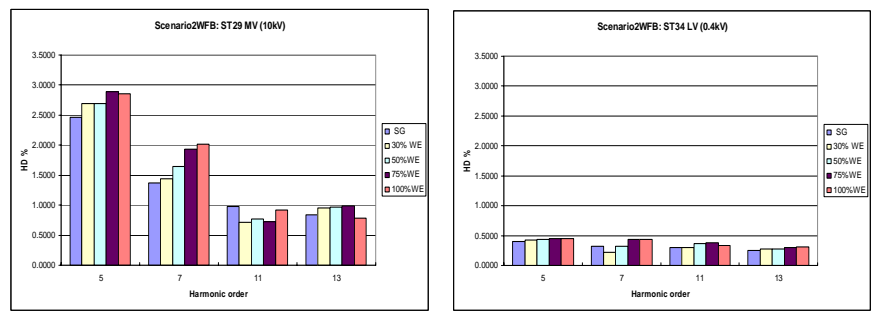

Fig. 10. Harmonic distortion on MV and Side "Scenario2_WFB"

Similar results compared to those of "Scenario1_WFA" are experienced in "Scenario2_WFB", but here the 5th harmonics distortion is more pronounced and the $11^{\text {th }}$ and $13^{\text {th }}$ harmonics show lesser distortion. It is thus assumed that this atypical harmonic distorting characteristic is created by some yet unidentified design parameter of the power system.

\section{Conclusion}

Based on this study, the impacts of the wind energy integration on power quality have been studied and the influence on the grid's power quality (Harmonic) of wind energy integration was measured on the low- and medium voltage level.

Although it is well-known that DFIG have a better influence on the power quality than other wind turbine generators and that DFIG characteristics introduce some improvements on the harmonics index results, it can be seen that the harmonic content delivers influence on the output power terms. This is demonstrated with the increase of 
THD\% and HD\% on the MV and LV side when more wind energy is integrated on the system.

The results up to this point demonstrate that the influence of increasing renewable energy generation weakens the system and render it vulnerable to power quality perturbations.

All procedures are being carried out with a model. Therefore it is necessary to take into account the limitations of the simulations and it will be interesting for future developments to compare the results with real case studies in order to prove the accuracy of the simulations The analysis made will be used for future research like a reference for different developments and solutions in the wind integration on networks with different voltage levels.

\section{References}

[1] Utility Wind Integration group, www.uwig.com.

[2] T. Ackermann, "Wind Power in Power Systems". Royal Institute of Technology. John Wiley and Sons Ltd., Sweden, 2005.

[3] DIgSILENT Power Factory Version 13.1.257, Educational Versions

[4] EN 50160. Voltage characteristics of electricity supplied by public distribution systems; German version EN 50160:199

[5] E.ON - Netz. \Grid Code - High and Extra High Voltage". August 2003. www.eon-netz.com

[6] Eping, J. Stenzel, M. Pöller, H. Müller, "Impact of Large Scale Wind Power on Power System Stability", 5th International Workshop on Large-Scale Integration of Wind Power and Transmission Networks for Offshore Wind Farms, Glasgow, Scotland 2005

[7] M. Pöller, S. Achilles, “Aggregated Wind Park Models for Analyzing Power System Dynamics". 4th International Workshop on Large-Scale Integration of Wind Power and Transmission Networks for Off-shore Wind Farms, Billund, Denmark 2003

\section{Biografies}

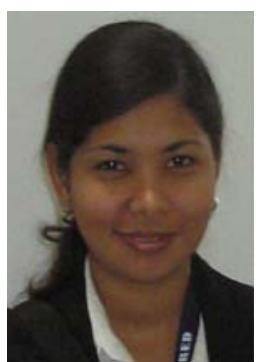

Elda Vilchez (1979) received Dipl.-Ing. degree in Electrical Engineering, Power System, from the University Bicentenaria of Aragua, Venezuela, in 2003, and her Master Degree in Electrical Power Engineering, from the Darmstadt University of Technology, Germany, in 2006. She is currently doing her $\mathrm{PhD}$ thesis at Darmstadt University of Technology, Institute of Electrical Energy Systems in the area of power quality. Her research interests are power quality, voltage sags, transmission system and wind integration.

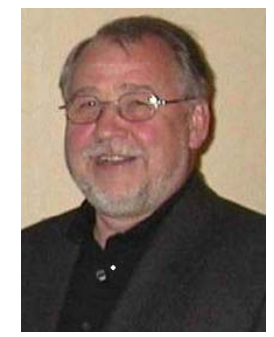

Juergen Stenzel (1944) received Dipl.-Ing. degree in Electrical Engineering, from Munich University of Technology, Germany, in 1970, and the Dr.-Ing. Degree from the University of Siegen, Germany, in 1984. Currently, he is in the Darmstadt University of Technology, Germany, where he received a full professorship in the Department of Electrical Engineering and Information Technology. He is a member of the Institute of Electrical Energy Systems. His area of interest is power system analysis and control. 